\title{
Características relaCiONADAS AO PRIMEIRO E Último PaRTO POR CESÁrea
}

\author{
Arlete Maria dos Santos Fernandes*, Aloísio José Bedone, larissa Capochin Paes leme, Gislaine Aparecida Fonsechi-Carvasan
}

Trabalho realizado na Área de Tocoginecologia, Faculdade de Ciências Médicas, Pontifícia Universidade Católica de Campinas, SP

\author{
*Correspondência: \\ Caixa postal 6181 \\ Campinas/SP \\ Cep: 13084-971 \\ Tel: (19) 3289-2856 \\ Fax: (19) $3289-2440$ \\ arlete@fcm.unicamp.br
}

\begin{abstract}
RESUMO
Oвjetivo. Estudar a associação entre a primeira e a última cesáreas e laqueadura tubária; determinar tempo de vida reprodutivo após o primeiro parto.

Métodos. Foram entrevistadas 653 mulheres com pelo menos duas gestações, de fevereiro a outubro de 2001 em hospital universitário, 172 com primeira e 294 com última cesárea. As variáveis foram sociodemográficas, obstétricas e características do primeiro e último partos e da laqueadura. Utilizou-se análise bivariada, posteriormente regressão múltipla com cálculo do odds ratio ajustado. Mulheres laqueadas foram separadas em grupos de 25 a 44 e $\geq 45$ anos, distribuídas em percentis e aplicou-se 0 teste de Wilcoxon para análise da idade na laqueadura e tempo de vida reprodutivo após o último parto. 0 estudo teve aprovação do Comitê de Ética.
\end{abstract}

RESULTADOS. Do total de mulheres, $89 \%$ tinham escolaridade $\leq 8$ anos e $78 \%$ eram brancas. Após regressão múltipla, associaram-se à primeira cesárea o último parto cesárea $(\mathrm{OR}=15,28, \mathrm{IC} 95 \%$ 8,54 a 27,36), ter companheiro $(\mathrm{OR}=3,87$, IC 95\% I,63 a 9, I7) e ter dado à luz nas décadas de 70, 80 e 90 (OR=4,43 IC 95\% I,37 a I4,27; OR=6, II, IC 95\% I,47 a 25,47; e OR=6,67, IC 95\% I,21 a 40,26), respectivamente. Estiveram associadas à última cesárea a laqueadura intraparto $(\mathrm{OR}=14,09, \mathrm{IC} 95 \%$ 7,37 a 26,97), ter dado à luz nas décadas de 70, 80 e 90 (OR=1,81, IC 95\% I,06 a 3,09; OR=5,53, IC $95 \%$ 3, I8 a 9,61; e OR=5,90, IC 95\% 3,03 a II,48), respectivamente, renda familiar > 5 salários $(\mathrm{OR}=2,41$, IC $95 \%$ I,42 a 4,08) e idade no primeiro parto $\geq 25$ anos (OR=I,80, IC $95 \%$ I,0I a 3,22). A idade média na laqueadura foi de 29 e 33,2 anos nas mulheres com 25 a 44 anos e $\geq 45$ anos, respectivamente $(p<0,001)$, o período reprodutivo após o primeiro parto foi de 9 e II,4 anos para os mesmos grupos $(p<0,001)$.

Conclusão. A primeira cesárea associou-se à última cesárea e esta se associou à laqueadura intraparto. A idade na laqueadura e a duraçãa do período de vida reprodutivo foram menores entre mulheres mais jovens.

UnITERMOS: Anticoncepção. Laqueadura tubáría. Cesárea.

\section{INTRODUÇÃO}

A prevalência de cesárea no Brasil gira em torno de 36\%, variando de $20 \%$ a $48 \%$ dos partos conforme a região'. Nos hospitais onde há predomínio de atendimento a pacientes conveniadas e particulares, as taxas de parto operatório chegam a superar $90 \%^{2}$. Esses números mostram-se muito elevados quando comparados com outros países. Segundo a Organização Mundial da Saúde (OMS), não há justificativa médica para taxas de cesárea maiores que 10\% a 15\%, já que países com as mais baixas taxas de mortalidade perinatal do mundo mantêm taxas menores que $10 \% 3$.

Dentre os fatores que contribuem para o elevado número de cesáreas no País, além do fator econômico e das cesáreas de repetição, a laqueadura tubária tem sido considerada como uma das causas importantes ${ }^{4}$. A prevalência da laqueadura entre mulheres brasileiras unidas, de 15 a 49 anos, é de 40\%, bastante superior aos países desenvolvidos ${ }^{5}$. Do ponto de vista da saúde pública, é imprescindível normatizar a indicação da laqueadura como método contraceptivo no Brasil. Entretanto, esta normatização tem se revelado um problema complexo. A prevalência da esterilização tubária varia, dependendo da região do País, dos índices de cesárea em cada região, da classe social da mulher e de fatores econômicos.
Com o objetivo de contribuir para o entendimento desse fenômeno social e médico, foi realizado estudo sobre a possível associação entre o tipo do primeiro e do último partos e algumas características sociodemográficas e obstétricas, em uma amostra de mulheres que freqüentaram hospital universitário. Foram estudadas associações entre cesárea no primeiro e último partos com a realização da laqueadura.

\section{Métodos}

Foi realizado estudo de coorte transversal com amostra de mulheres que freqüentaram o Ambulatório de Ginecologia Geral em serviço público universitário. Tomando por base a percentagem nacional de partos por cesárea (36\%), a prevalência de mulheres laqueadas (40\%) e o valor necessário para detectar-se diferenças entre as duas proporções de 12\%, calculamos amostra de 172 mulheres com parto cesárea, levando-se em conta erro alfa de 0,05 e beta de 0,20.

A diferença de $12 \%$ foi proposta por proporcionar a prevalência uma vez maior do número médio de cesáreas admitido como adequado pela OMS, entre 10\% e 15\%3.

Utilizou-se um questionário com perguntas fechadas, pré-testado e corrigido, para entrevista de mulheres que aguardavam consulta em Ambulatório de Ginecologia Geral, no período de fevereiro a outubro 
Fernandes AMS et AL.

de 200I. Foram incluídas mulheres com antecedente de pelo menos duas gestações e que aceitaram participar do estudo, às quais foi aplicado questionário estruturado sempre pela mesma entrevistadora. As variáveis estudadas foram idade da mulher no momento da entrevista (categorizada em $\leq 29$ anos, 30 a 39, 40 a 49, 50 a 59 e $\geq 60$ anos), raça (branca ou não branca), escolaridade ( $\leq 8$ e $>8$ anos), renda familiar ( $\leq 2,3$ a 5 e $>5$ salários mínimos), companheiro atual (sim/não), número de gestações ( 2,3 a 5 e $>6$ ), número de filhos vivos $(\leq 2$ e $\geq 3)$, idade no primeiro parto ( $\leq 19,20$ a 24 e $\geq 25$ anos), década do primeiro parto (anos 50, 60, 70, 80 ou 90), estar laqueada ou não, idade na laqueadura, laqueadura intraparto (sim/não), intervalo entre primeiro parto e laqueadura (anos), intervalo entre último parto e laqueadura (anos), categoria da internação hospitalar para realização da laqueadura (universitário, SUS e particular/convênio).

Inicialmente utilizou-se regressão bivariada com cálculo do odds ratio bruto e respectivo intervalo de confiança para cada variável estudada e, posteriormente, a regressão múltipla com cálculo do odds ratio ajustado e respectivo intervalo de confiança de $95 \%$. Para análise em regressão múltipla da variável independente "tipo de primeiro parto", foram consideradas todas as variáveis estudadas e, para segunda variável independente, "tipo de último parto", foram consideradas as variáveis escolaridade, renda, idade da mulher no primeiro parto, década do primeiro parto, estar laqueada e laqueadura intraparto.

O tipo de último parto foi analisado separadamente para a população de mulheres laqueadas, considerando-se as variáveis intervalo primeiro parto e laqueadura, intervalo último parto e laqueadura e tipo de internação para laqueadura. Para se avaliar a idade da mulher na laqueadura e o tempo de vida reprodutivo após o último parto, as mulheres laqueadas foram separadas em dois grupos de idade: 25 a 44 anos (ainda em fase reprodutiva) e $\geq 45$ anos (fase de climatério/ menopausa), tendo sido distribuídas em percentis e aplicado o teste de Wilcoxon' ${ }^{6}$. O estudo teve aprovação do Comitê de Ética da PUCCampinas.

\section{Resultados}

Foram entrevistadas 653 mulheres, sendo que 418 (64\%) tinham idade até 49 anos e 235 (36\%) tinham 50 anos ou mais; os extremos de idade foram 25 e 82 anos. Haviam tido primeiro parto cesárea e vaginal, respectivamente, 172 e 48 I mulheres. A análise das variáveis estudadas em relação ao tipo de primeiro parto encontra-se na Tabela I.

Mais de dois terços das mulheres eram brancas, a maior parte de ambos os grupos tinha até oito anos de escolaridade. Entre as mulheres que tiveram o primeiro parto por cesárea, a maioria teve um último parto também por cesárea (87\%), enquanto entre as mulheres que haviam tido primeiro parto via vaginal somente $30 \%$ foram submetidas à cesárea no último parto.

Pela regressão bivariada, encontrou-se que ter maior escolaridade e renda familiar, viver com o companheiro, ter menor número de filhos vivos, o último parto por cesárea, idade superior a 25 anos no primeiro parto e ter dado à luz nas últimas três décadas foram as variáveis relacionadas ao primeiro parto por cesárea. Após ajuste do cálculo do odds ratio para todas as variáveis, restaram diretamente associadas à primeira cesárea o último parto por cesárea, ter dado à luz nas últimas três décadas e ter um companheiro; ao contrário, associação inversa foi observada nas mulheres com seis filhos vivos ou mais (Tabela I).

A mesma análise foi realizada em relação ao tipo de último parto (Tabela 2). Haviam tido último parto por cesárea 294 mulheres e via vaginal 359 mulheres. Verificou-se, na análise bivariada, que a escolaridade maior que 8 anos, a elevação progressiva da renda familiar, idade no primeiro parto igual ou superior a 25 anos, ter dado à luz nas últimas três décadas, estar laqueada e ter realizado laqueadura intraparto estiveram associadas ao último parto por cesárea. Após o ajuste para as demais variáveis, restaram diretamente relacionadas ao último parto por cesárea a maior renda, idade no primeiro parto de 25 anos ou mais, ter dado à luz nas últimas três décadas e ter realizado a laqueadura intraparto (Tabela 2).

Para a população de mulheres laqueadas foi avaliado o tipo de último parto em relação às características da laqueadura (Tabela 3). A análise bivariada mostrou que a cesárea no último parto esteve associada às seguintes características da laqueadura: realização durante 0 parto, durante internação por convênio ou particular, e período menor que dez anos de vida reprodutiva após o primeiro parto. Após regressão múltipla, as variáveis que restaram associadas à cesárea no último parto foram períodos de vida reprodutiva após o primeiro parto menores que dez anos (Tabela 3).

As mulheres laqueadas foram separadas em dois grupos de idade, com 25 a 44 anos e $\geq 45$ anos, e distribuídas em percentis conforme a média de idade no momento da laqueadura e o período decorrido entre o primeiro parto e a laqueadura, em anos (Tabela 4). A análise mostrou diferença para as duas variáveis nos dois grupos. Tanto o intervalo de vida reprodutiva após o primeiro parto foi, em média, dois anos menor no grupo de mulheres mais jovens, como a idade da mulher no momento da laqueadura foi em média quatro anos menor entre as mulheres de menor idade (Tabela 4).

\section{Discussão}

Neste estudo, foi possível observar que a primeira cesárea associou-se ao último parto também cesárea e este associou-se à realização de laqueadura intraparto. No grupo de mulheres em fase de vida reprodutiva, houve diminuição da idade na laqueadura e do intervalo entre o primeiro parto e a laqueadura, em relação ao grupo de mulheres com 45 anos ou mais.

Estes resultados corroboram os dados previamente conhecidos da atenção obstétrica dispensada nas últimas décadas no Brasil. A tendência de que após um primeiro parto operatório, o parto subseqüente seja também operatório foi anteriormente observada ${ }^{4}$; entretanto, é difícil determinar o quanto a cesárea pode estar contribuindo para limitar a prole dos casais, já que existem fatores culturais e de transformação social que influenciaram o número de filhos da população nas últimas décadas. A maior instrução da mulher, sua maior inserção no mundo do trabalho e a opção pela melhoria da qualidade de vida familiar são transformações que levaram à diminuição da fecundidade no Brasil. A Pesquisa Nacional de Demografia e Saúde (PNDS) mostrou taxa global de fertilidade de 2,5 filhos/mulher, sendo maior para a população rural (3,5 filhos/mulher) que para a urbana (2,3 filhos/ mulher), ambas apresentando declínio claro nos últimos anos ${ }^{5}$. 
Tabela I - Análise bivariada e regressão múltipla das mulheres com primeiro parto cesárea e vaginal segundo as características estudadas

\begin{tabular}{|c|c|c|c|c|}
\hline & \multicolumn{2}{|c|}{ Tipo de primeiro parto } & \multirow[b]{2}{*}{$\begin{array}{c}\text { Odds ratio } \\
\text { bruto (IC 95\%) }\end{array}$} & \multirow[b]{2}{*}{$\begin{array}{c}\text { Odds ratio } \\
\text { ajustado* (IC } 95 \%)\end{array}$} \\
\hline & $\begin{array}{l}\text { Cesárea } \\
(\mathrm{n}=172)\end{array}$ & $\begin{array}{l}\text { Vaginal } \\
(n=48 I)\end{array}$ & & \\
\hline \multicolumn{5}{|l|}{ Idade } \\
\hline Até 29 anos & $18(10,5)$ & $39(8,1)$ & ref & ref \\
\hline 30 a 39 anos & $65(37,8)$ & $92(19,1)$ & NS & NS \\
\hline 40 a 49 anos & $58(33,7)$ & $146(30,4)$ & NS & NS \\
\hline 50 a 59 anos & $27(15,7)$ & $112(23,3)$ & NS & NS \\
\hline$\geq 60$ anos & $4(2,3)$ & $92(19,1)$ & $0,09(0,03-0,30)$ & NS \\
\hline \multicolumn{5}{|l|}{ Raça } \\
\hline Branca & $139(80,8)$ & $37 \mid(77,1)$ & ref & ref \\
\hline Não branca & $33(19,2)$ & $110(22,9)$ & NS & NS \\
\hline \multicolumn{5}{|l|}{ Escolaridade } \\
\hline$\leq 8$ anos & $139(80,8)$ & $447(92,9)$ & ref & ref \\
\hline$>8$ anos & $33(19,2)$ & $34(7,1)$ & $3,12(1,86-5,23)$ & NS \\
\hline \multicolumn{5}{|l|}{ Renda } \\
\hline$\leq 2 \mathrm{SM}$ & $24(14,0)$ & $176(36,6)$ & ref & ref \\
\hline 3 a 5 SM & $82(47,7)$ & $181(37,6)$ & $3,32(2,02-5,48)$ & NS \\
\hline$>5 S M$ & $66(38,4)$ & $124(25,8)$ & $3,90(2,32-6,57)$ & NS \\
\hline \multicolumn{5}{|c|}{ Companheiro atual } \\
\hline Sim & $163(94,8)$ & $378(78,6)$ & $4,94(2,44-9,99)$ & $3,87(1,63-9,17)$ \\
\hline Não & $9(5,2)$ & $103(21,4)$ & ref & ref \\
\hline \multicolumn{5}{|c|}{ Gestaçôes anteriores } \\
\hline$=2$ & $63(36,6)$ & $82(17,0)$ & ref & ref \\
\hline 3 a 5 & $99(57,6)$ & $252(52,4)$ & $0,51(0,34-0,76)$ & NS \\
\hline$\geq 6$ & $10(5,8)$ & $147(30,6)$ & $0,09(0,04-0,18)$ & $0,26(0,09-0,76)$ \\
\hline \multicolumn{5}{|l|}{ Filhos vivos } \\
\hline$\leq 2$ & $83(48,3)$ & $125(26,0)$ & $2,66(1,85-3,81)$ & NS \\
\hline$\geq 3$ & $89(51,7)$ & $356(74,0)$ & ref & ref \\
\hline \multicolumn{5}{|c|}{ Último parto } \\
\hline Vaginal & $22(12,8)$ & $337(70,1)$ & ref & ref \\
\hline Cesárea & $150(87,2)$ & | $44(29,9)$ & $15,96(9,79-26,00)$ & $15,28(8,54-27,36)$ \\
\hline \multicolumn{5}{|c|}{ Idade no primeiro parto } \\
\hline$\leq 19$ anos & $54(31,4)$ & $20 \mid(4 \mid, 8)$ & ref & ref \\
\hline 20 a 24 anos & $72(41,9)$ & $219(45,5)$ & NS & NS \\
\hline$\geq 25$ anos & $46(26,7)$ & $61(12,7)$ & $2,81(1,73-4,57)$ & NS \\
\hline \multicolumn{5}{|c|}{ Década do primeiro parto } \\
\hline Anos 50 & I $(0,6)$ & $72(15,0)$ & ref & ref \\
\hline Anos 60 & $8(4,7)$ & $106(22,0)$ & ref & ref \\
\hline Anos 70 & $50(29,1)$ & $148(30,8)$ & $6,68(3,18-14,03)$ & $4,43(1,37-14,27)$ \\
\hline Anos 80 & $80(46,5)$ & $107(22,2)$ & $14,78(7,13-30,66)$ & $6,11(1,47-25,47)$ \\
\hline Anos 90 & $33(19,2)$ & $48(10,0)$ & $13,59(6,09-30,34)$ & $6,67(1,21-40,26)$ \\
\hline \multicolumn{5}{|c|}{ Estar laqueada } \\
\hline $\operatorname{Sim}$ & $99(57,6)$ & $236(49,1)$ & NS & NS \\
\hline Não & $73(42,4)$ & $245(50,9)$ & ref & ref \\
\hline
\end{tabular}

*Regressão mútipla considerando todas as variáveis; IC: intervalo de confiança; SM: salário mínimo (correspondia a R\$137,00) 


\begin{tabular}{|c|c|c|c|c|}
\hline & \multicolumn{2}{|c|}{ Último parto } & \multirow[b]{2}{*}{$\begin{array}{c}\text { Odds ratio } \\
\text { bruto (IC } 95 \%)\end{array}$} & \multirow[b]{2}{*}{$\begin{array}{c}\text { Odds ratio } \\
\text { ajustado* (IC } 95 \%)\end{array}$} \\
\hline & $\begin{array}{l}\text { Cesárea } \\
(n=294)\end{array}$ & $\begin{array}{l}\text { Vaginal } \\
(n=359)\end{array}$ & & \\
\hline \multicolumn{5}{|l|}{ Escolaridade } \\
\hline$<8$ anos & $249(84,7)$ & $337(93,9)$ & ref & ref \\
\hline$>8$ anos & $45(15,3)$ & $22(6,1)$ & $2,77(1,62-4,73)$ & NS \\
\hline \multicolumn{5}{|l|}{ Renda } \\
\hline$\leq 2 S M$ & $59(20,1)$ & $|4|(39,3)$ & ref & ref \\
\hline 3 a 5 SM & $126(42,9)$ & $137(38,2)$ & $2,20(1,49-3,24)$ & $2,05(1,27-3,30)$ \\
\hline$>5 S M$ & $109(37,1)$ & $81(22,6)$ & $3,22(2,12-4,89)$ & $2,41(1,42-4,08)$ \\
\hline \multicolumn{5}{|c|}{ Idade no primeiro parto } \\
\hline$\leq 19$ anos & $109(37,1)$ & $146(40,7)$ & ref & ref \\
\hline 20 a 24 anos & $120(40,8)$ & $17 \mid(47,6)$ & NS & NS \\
\hline$\geq 25$ anos & $65(22,1)$ & $42(\mid 1,7)$ & $2,07(1,31-3,29)$ & $1,80(1,01-3,22)$ \\
\hline \multicolumn{5}{|c|}{ Década do primeiro parto } \\
\hline Anos 50 & $4(1,4)$ & $69(19,2)$ & ref & ref \\
\hline Anos 60 & $37(12,6)$ & $77(21,4)$ & ref & ref \\
\hline Anos 70 & $96(32,7)$ & $102(28,4)$ & $3,35(2,15-5,23)$ & $|, 8|(1,06-3,09)$ \\
\hline Anos 80 & $116(39,5)$ & $71(19,8)$ & $5,82(3,69-9,17)$ & $5,53(3,18-9,61)$ \\
\hline Anos 90 & $4 \mid(\mid 3,9)$ & $40(\mid 1,1)$ & $3,65(2,09-6,37)$ & $5,90(3,03-11,48)$ \\
\hline \multicolumn{5}{|c|}{ Estar laqueada } \\
\hline Não & $89(30,3)$ & $229(63,8)$ & ref & ref \\
\hline Sim & $205(69,7)$ & $130(36,2)$ & $4,06(2,92-5,64)$ & NS \\
\hline \multicolumn{5}{|c|}{ Laqueadura intraparto } \\
\hline Não & $107(36,4)$ & $301(83,8)$ & ref & ref \\
\hline Sim & $187(63,6)$ & $58(16,2)$ & $9,07(6,28-13,10)$ & |4,09(7,37-26,97) \\
\hline
\end{tabular}

*Regressão mútipla considerando as variáveis escolaridade, renda, idade da mulher no primeiro parto, década do primeiro parto, estar laqueada e laqueadura intraparto

Observou-se associação direta entre o tipo do primeiro e do último partos. Quando o primeiro parto foi cesárea, $87 \%$ das mulheres apresentaram último parto também cesárea, enquanto que, quando o primeiro parto foi vaginal, em 70\% dos casos o último parto foi também vaginal. Este fato chama atenção para a indicação da via abdominal na primeira parturição, que poderá influenciar claramente a atenção obstétrica futura. $\mathrm{Na}$ tentativa de diminuir o número de cesáreas, a escola norte-americana que, até os anos 70 , seguia a prática de que "uma vez cesárea sempre cesárea" concluiu recentemente, após analisar o período de 1989 a 1999, comparando resultados perinatais e maternos, que a prova de trabalho de parto é opção razoável para mulheres com uma cesárea anterior?

Apesar da conhecida relação entre a cesárea e a maior escolaridade e renda ${ }^{4,8}$, neste estudo não foi possível detectar diferenças entre grau de instrução da mulher e partos por cesárea, possivelmente devido ao tipo de clientela estudada. Nos dados da PNDS, a cesárea mostrou forte associação com o nível de instrução de mulheres que haviam dado à luz entre os anos 1992 e 1996, aumentando progressivamente com o número de anos de estudo da mulher. Mulheres sem escolaridade, com escolaridade de 4 a 8 anos, 9 a II e com 12 anos ou mais tiveram taxas de cesárea de $12,5 \%, 37 \%, 54,6 \%$ e $81,3 \%$, respectivamente ${ }^{5}$. Nos dados do município de Campinas referentes aos primeiros sete meses de 2005, observou-se que, quando o grau de instrução da mãe variou de menos de 8 anos, 8 a II anos e maior de 12 anos, houve elevação da prevalência de cesárea de $50,8 \%, 62,2 \%$ e 88,5\%, respectivamente².
A prevalência de último parto por cesárea no total de mulheres entrevistadas neste estudo foi de 45\%, similar à prevalência no País. Entre mulheres que deram à luz de 1992 a 1996, tiveram cesárea $36,4 \%$, sendo $41,8 \%$ residentes em área urbana e 20,1\% em área rural ${ }^{5}$. Embora o atendimento em hospitais de convênio e particulares apresente maiores taxas de cesárea, nesta amostra de mulheres com características de baixa renda e escolaridade, composta usualmente de clientela do Sistema Único de Saúde (SUS), o número de cesáreas foi alto. Em dados do município de Campinas referentes ao ano de 2002, houve atendimento a 19.015 nascidos vivos, II.277 (59,3\%) deles nascidos por cesárea. A prevalência de cesárea segundo o tipo de estabelecimento hospitalar foi de 38\% nos hospitais universitários, $61 \%$ nos hospitais que atendem clientela mista, ou seja, pacientes particulares, de convênio e do SUS, e 88\% naqueles cujo atendimento é exclusivamente para conveniados e particulares ${ }^{2}$.

No Brasil, o parto cesárea tem sido considerado como o de melhor qualidade de cuidado médico e o fato das classes mais abastadas terem maior acesso ao parto eletivo faz com que muitas mulheres com menor aporte econômico também busquem esse tipo de atendimento, o avaliem como de melhor qualidade e o desejem?. Entretanto, estudos recentes não têm dado suporte à crença de que o aumento do número de cesáreas possa ser explicado pelo desejo das mulheres de escolher o parto operatório ${ }^{10,11}$ e fatores não obstétricos têm sido cogitados como influenciadores das indicações de cesárea pelos médicos ${ }^{12}$. 
Tabela 3 - Análise bivariada e regressão múltipla das mulheres laqueadas com último parto cesárea e vaginal segundo as características relacionadas à laqueadura

\begin{tabular}{|c|c|c|c|c|}
\hline & \multicolumn{2}{|c|}{ Último parto } & \multirow[b]{2}{*}{$\begin{array}{l}\text { Odds ratio } \\
\text { bruto (IC 95\%) }\end{array}$} & \multirow[b]{2}{*}{$\begin{array}{c}\text { Odds ratio } \\
\text { ajustado* (IC 95\%) }\end{array}$} \\
\hline & $\begin{array}{l}\text { Cesárea } \\
(n=205)\end{array}$ & $\begin{array}{l}\text { Vaginal } \\
(n=\mid 30)\end{array}$ & & \\
\hline \multicolumn{5}{|c|}{ Intervalo primeiro parto e laqueadura } \\
\hline $\begin{array}{l}\leq 5 \text { anos } \\
6 \text { a } 10 \text { anos }\end{array}$ & $\begin{array}{l}58(28,3) \\
81(395)\end{array}$ & $\begin{array}{l}20(15,4) \\
37(28,5)\end{array}$ & $\begin{array}{l}3,21(1,75-5,89) \\
242(145-404)\end{array}$ & $\begin{array}{l}3,41(1,54-7,54) \\
2,57(1,39-4,75)\end{array}$ \\
\hline$\geq \|$ anos & $66(32,2)$ & $73(56,2)$ & $\begin{array}{l}\text { ref } \\
\text { ref }\end{array}$ & $\begin{array}{l}\text { ref } \\
\text { ref }\end{array}$ \\
\hline \multicolumn{5}{|c|}{ Intervalo último parto e laqueadura } \\
\hline Laqueadura no último parto & $187(91,2)$ & $58(44,6)$ & $7,88(3,44-18,07)$ & NS \\
\hline I a 5 anos & $9(4,4)$ & $50(38,5)$ & NS & NS \\
\hline$\geq 6$ anos & $9(4,4)$ & $22(16,9)$ & ref & ref \\
\hline \multicolumn{5}{|c|}{ Tipo de internação para laqueadura } \\
\hline Hospital Universitário & $18(8,8)$ & $22(16,9)$ & ref & ref \\
\hline SUS & $99(48,3)$ & $79(60,8)$ & NS & NS \\
\hline Convênio/particular & $88(42,9)$ & $29(22,3)$ & 3,7I (I,75-7,86) & NS \\
\hline
\end{tabular}

*Regressão múltipla considerando as variáveis intervalo primeiro parto e laqueadura, intervalo último parto e laqueadura e tipo de internação para laqueadura; SUS: Sistema Único de Saúde (setor público)

\begin{tabular}{|c|c|c|c|c|c|c|c|c|c|}
\hline Idade & \multicolumn{9}{|c|}{ Tempo entre primeiro parto e laqueadura (anos) } \\
\hline \multirow[t]{2}{*}{$\geq 45$ anos } & $\begin{array}{l}153 \\
182\end{array}$ & $\begin{array}{l}9,03 \\
11,43\end{array}$ & $\begin{array}{l}, 82 \\
6,53\end{array}$ & $\begin{array}{l}5,00 \\
6,00\end{array}$ & $\begin{array}{c}8,00 \\
10,50\end{array}$ & $\begin{array}{l}12,00 \\
15,00\end{array}$ & $\begin{array}{l}1,00 \\
1,00\end{array}$ & $\begin{array}{l}26,00 \\
30,00\end{array}$ & $<0,001$ \\
\hline & $\mathrm{n}$ & média & desvio padrão & percentil 25\% & mediana & percentil 75\% & mínimo & máximo & $\mathrm{p}^{*}$ \\
\hline $\begin{array}{l}25 \text { a } 44 \text { anos } \\
\geq 45 \text { anos }\end{array}$ & $\begin{array}{l}153 \\
182\end{array}$ & $\begin{array}{l}29,01 \\
33,24\end{array}$ & $\begin{array}{l}5,00 \\
5,66\end{array}$ & $\begin{array}{l}26,00 \\
29,00\end{array}$ & $\begin{array}{l}28,00 \\
33,00\end{array}$ & $\begin{array}{l}32,00 \\
37,00\end{array}$ & $\begin{array}{l}18,00 \\
18,00\end{array}$ & $\begin{array}{l}49,00 \\
49,00\end{array}$ & $<0,001$ \\
\hline
\end{tabular}

*Teste de Wilcoxon

A realização da laqueadura intraparto tem sido apontada como um dos fatores que contribuíram para o aumento dos partos operatórios nas últimas décadas ${ }^{4}$. Essa distorção foi responsável pela proibição da esterilização no momento do parto como critério para aplicação da lei sobre o tema. Tal determinação, no entanto, levou a um viés que também não tem sido benéfico para as mulheres que não podem ser esterilizadas no momento do parto. A única alternativa deixada pela lei à viabilização do procedimento intraparto foi no caso de cesáreas repetidas, o que também pode permitir o vício da implementação do parto por cesárea para permitir a esterilização.

Acontecimentos que permearam o atendimento obstétrico brasileiro nas últimas décadas foram os maiores determinantes da atual distorção da atenção aos nascimentos na rede hospitalar. Foi importante distribuir as mulheres laqueadas em duas faixas de idade, sob pena de não detectarmos variações na atenção nos diferentes períodos. Nesta amostra de mulheres de baixa escolaridade e renda, o período de vida reprodutivo após o primeiro parto foi menor nas mulheres de menor faixa etária, o mesmo ocorrendo com a idade no momento de realização da laqueadura. Apesar da dificuldade de se avaliar a associação entre o tipo de primeiro parto e a duração do período reprodutivo da mulher, certamente a associação existe e é previsível no momento da indicação da cesárea como via do primeiro parto, especialmente entre as mulheres jovens.

\section{Conclusão}

Este estudo buscou aproximação entre algumas características sociodemográficas e da atenção obstétrica dispensada a mulheres nas últimas décadas, com as prevalências de cesárea e laqueadura. Outros estudos devem ser realizados para o acompanhamento desse processo para que possamos criar medidas de correção, abrangência e qualidade de atenção para toda a população em idade reprodutiva. O acompanhamento das mudanças no atendimento nas áreas da reprodução deve continuar e talvez nas próximas décadas seja possível detectar maior qualidade no serviço prestado nessa área da atenção médica se hoje nos dispusermos a capacitar e educar de forma crítica os novos profissionais que serão inseridos no mercado de trabalho. 
Fernandes AMS et AL.

\section{Conflito de interesse: não há.}

\section{SUMMARY}

Characteristics related to the first and last cesarean delivery among Women from a Campinas University Hospital

OBIECTIVE. To study the association between first and last caesarian sections with tubal sterilization; to determine length of reproductive life after the first delivery.

METHODS. From February to October 200 I in a university hospital, interviews were carried out with 653 women having had at least two pregnancies. Of these women, 172 had a first caesarian section; 294 had a last caesarian section. Variables were social demographic characteristics, obstetric history and characteristics of the first and last deliveries and tubal sterilization. Bivariate analysis was performed, followed by multiple regression analysis calculating the adjusted odds ratio. Women who had undergone tubal sterilization were divided into age groups of 25 to 44 and $\geq 45$ years in a percentile distribution. The Wilcoxontest was used to analyze age at tubal sterilization and length of reproductive life after the last delivery. The study was approved by the Ethics Committee.

RESULTS. Of these women, $89 \%$ completed $\leq 8$ years of school education and $78 \%$ were Caucasian. On multiple regression analysis, there was an association between the first and last caesarian section $(O R=15.28,95 \% \mathrm{Cl} 8.54$ to 27.36), having a partner $(O R=3.87, \mathrm{C} 195 \%$ 1.63 to 9.17$)$ and giving birth in the '70s, '80 or ' $90 \mathrm{~s}(\mathrm{OR}=4.43,95 \% \mathrm{Cl}$ 1.37 to 14.27), $(O R=6.11,95 \% \mathrm{Cl} 1.47$ to 25.47$)$ and $(O R=6.67$, $95 \% \mathrm{Cl} 1.21$ to 40.26$)$, respectively. The last caesarian section was associated with intrapartum tubal sterilization $(O R=14.09,95 \% \mathrm{Cl} 7.37$ to 26.97), giving birth in the '70s, '80s or ' $90 \mathrm{~s}(\mathrm{OR}=1.81,95 \% \mathrm{Cl} 1.06$ to 3.09), $(O R=5.53,95 \% \mathrm{Cl} 3.18$ to 9.61$)$ and $(O R=5.90,95 \% \mathrm{Cl} 3.03$ to 11.48$)$, respectively, family income of $>5$ minimum wages $(O R=2.41$, $95 \% \mathrm{Cl} 1.42$ to 4.08$)$ and age at first delivery $\geq 25$ years $(O R=1.80$, $95 \% \mathrm{Cl} 1.01$ to 3.22). Mean age at sterilization was 29.0 and 33.2 years in women aged 25 to 44 years and $\geq 45$ years, respectively $(p<0.001)$. The duration of the reproductive period after the first delivery was 9.0 and II.4 years for the same groups $(p<0.001)$.

CONCLUSION. The first caesarian section was associated with the last caesarian section. The last caesarian section was associated with intrapartum tubal sterilization. Age at sterilization was lower and the reproductive period was shorteramongyoungerwomen. [RevAssoc Med Bras 2007; 53(I): 53-8]

KEY wORDS: Contraception. Tubal sterilization. Cesarean delivery.

\section{REFERÊNCIAS}

I. Ministério da Saúde. Anuário Estatístico de Saúde do Brasil, 200 I . [citado 2006 jun 16]. Disponível em: http://portal.saude.gov.br/portal/ aplicações/anuário200 I/index.cfm.

2. SINASC. Sistema de Informação TabNet. Secretaria Municipal de Campinas. [citado 2006 jun 16]. Disponível em: http:// tabnet.saude.campinas.sp.gov.br/cgi/tabcgi.exe?sinasc/Nascamp.def.

3. World Health Organization. Appropriate technology for birth. Lancet. 1 985;2:436-7.

4. Barros FC, Vaughan JP, Victora CG, Huttly SRA. Epidemic of cesarean sections in Brazil. Lancet. 1991;338:167-69.

5. Pesquisa Nacional sobre Demografia e Saúde. PNDS 1996. Rio de Janeiro: BENFAM; 1997.

6. Armitage P, Berry G. Statistical methods in medical research. $2^{\text {nd }}$ ed. Oxford: Blackwell Scientific Publications; 1985.

7. Mozurkewich EL, Hutton EK. Elective repeat cesarean delivery versus trial of labor: a meta-analysis of the literature from 1989 to 1999. Am J Obstet Gynecol. 2000; 183: I 187-97.

8. Freitas PF, Drachler M de L, Leite JC, Grassi PR. Social inequalities in cesarean section rates in primiparae, southern Brazil. Rev Saúde Pública. 2005;39:761-7.

9. Béhague DP, Victora CG, Barros FC. Consumer demand for caesarean sections in Brazil: population based birth cohort study linking ethnographic and epidemiological methods. BMJ. 2002;324:942-5.

10. Faundes A, Pádua KS, Osis MJ, Cecatti JG, Sousa MH. Brazilian women and physicians' viewpoints on their preferred route of delivery. Rev Saúde Pública. 2004;38:488-94.

I I. Barbosa GR, Giffin K, Angulo-Tuesta A, Gama A de S, Chor D, D'Orsi E, et al. Cesarean sections: who wants them and under what circumstances? Cad Saúde Pública. 2003; 19:1611-20.

12. Dias MA, Deslandes SF. Cesarean sections: risk perception and indication by attending obstetricians in a public maternity hospital in Rio de Janeiro. Cad Saúde Pública. 2004;20: 109-16. Aceito para publicação: 15/08/06 